\title{
Ion induced changes in the structure of bordered pit membranes
}

\author{
Jinkee Lee ${ }^{1}$, N. Michele Holbrook ${ }^{2}$ and Maciej A. Zwieniecki ${ }^{3}{ }^{*}$ \\ ' School of Mechanical Engineering, Sungkyunkwan University, Suwon, South Korea \\ 2 Department of Organismic and Evolutionary Biology, Harvard University, Cambridge, MA, USA \\ ${ }^{3}$ The Arnold Arboretum, Harvard University, Boston, MA, USA
}

\section{Edited by:}

Peter Jegsen Melcher, Ithaca College, USA

\section{Reviewed by:}

Ingo Dreyer, University of Potsdam, Germany

Lars Hendrik Wegner, Karlsruhe Institute of Technology, Germany

*Correspondence:

Maciej A. Zwieniecki, The Arnold Arboretum, Harvard University,

Boston, MA 02131, USA

e-mail:mzwienie@oeb.harvard.edu
Ion-mediated changes in xylem hydraulic resistance are hypothesized to result from hydrogel like properties of pectins located in the bordered pit membranes separating adjacent xylem vessels. Although the kinetics of the ion-mediated changes in hydraulic resistance are consistent with the swelling/deswelling behavior of pectins, there is no direct evidence of this activity. In this report we use atomic force microscopy (AFM) to investigate structural changes in bordered pit membranes associated with changes in the ionic concentration of the surrounding solution. When submerged in de-ionized water, AFM revealed bordered pit membranes as relatively smooth, soft, and lacking any sharp edges surface, in contrast to pictures from scanning electron microscope (SEM) or AFM performed on air-dry material. Exposure of the bordered pit membranes to $50 \mathrm{mM} \mathrm{KCl}$ solution resulted in significant changes in both surface physical properties and elevation features. Specifically, bordered pit membranes became harder and the fiber edges were clearly visible. In addition, the membrane contracted and appeared much rougher due to exposed microfibers. In neither solution was there any evidence of discrete pores through the membrane whose dimensions were altered in response to the ionic composition of the surrounding solution. Instead the variable hydraulic resistance appears to involve changes in the both the permeability and the thickness of the pit membrane.

Keywords: bordered pit membrane, xylem, pectin, hydraulic resistance, atomic force microscopy

\section{INTRODUCTION}

Self-actuating hybrid materials are used in a wide range of microdevices ranging from flux control in microfluidic systems using gels (Beebe et al., 2000) to shape memory actuators (Vyawahare et al., 2008). The growing interest in the use of "smart materials" results from the fact that such materials do not require external triggers, rather they simply respond to changes in their surroundings. Such autonomously acting materials occur within the plant vascular network. Trees must move large volumes of water from soil to atmosphere to sustain photosynthetic activity. Because the supply of water in terrestrial ecosystems is often limiting, the ability to tune the distribution of water across plant's dendritic structure may play an important role in optimization of resource use (Holbrook and Zwieniecki, 2005; Zwieniecki and Holbrook, 2009; Shackel et al., 2010). The movement of water through the plant vascular system occurs via specialized micro-channels composed of dead cells arranged in series. The ability of these cells to respond to the ionic concentration in the transpiration stream allows the seemingly dead xylem micro-channels to modulate their hydraulic properties (Zwieniecki et al., 2001). Bordered pit membranes create a porous boundary between adjacent micro-channels, that prevents the spread of embolism that could halt water transport from soil to leaves and ultimately cause plant death. Pit membranes are a composite material that includes cellulose, lignin, hemicelluloses, and pectin hydrogels (Zwieniecki and Holbrook, 2000; Liu et al., 2004). It also provides a significant hydraulic resistance to xylem sap flow that was shown to be variable in response to sap ion concentration.

Research on the structure and function of bordered pit membranes most often consists of either the indirect study of their properties by inferring them from analysis of liquid fluxes across plant stem segments (Zwieniecki et al., 2001) or scanning electron microscope (SEM) observation of dry plant material (Choat et al., 2006). This comes from the fact that bordered pit membranes are very small ( $\sim 5 \mu \mathrm{m}$ diameter) and hidden among overarching heavy cellulose-lignin walls that prevent easy access to membrane surface. More importantly, bordered pit membranes in vivo are immersed in water and in an intact plant, cations in the surrounding solution "open" the bordered pit membrane, facilitating the movement of water. This observed hydraulic activity is the bases for the hydrogel hypothesis that the positive ion shrinks the hydrogel (pectin) covering the nanofabrils of membrane changing its porosity. However, hydraulic measurements do not provide direct evidence of physical bases for the altered flow resistance change. SEM makes it possible to image the nano-fibers but this is usually done using imprints or visualizing dried samples imaged in high vacuum (Jansen et al., 2009). Using environmental SEM it is possible to image bordered pit membranes in humid air at some level of hydration but never under water and never with the resolution needed (Kirk et al., 2009). Recently, new insights into bordered pit membrane structural properties have been obtained using atomic force microscopy (AFM) on air-dry material (Pesacreta et al., 2005; 
Dute and Elder, 2011). The obvious next step is to use liquid phase AFM to investigate how the structure of bordered pit membranes changes in response to the ionic composition of surrounding solution and what properties of this composite material are responsible for the changes in its hydraulic properties.

\section{MATERIALS AND METHODS SAMPLE PREPARATION}

Tobacco plants (Nicotiana tabacum L.) were grown in a greenhouse under natural light conditions until stem diameters exceeded $10 \mathrm{~mm}$. A 5-cm-long stem segment was excised and the xylem sectioned longitudinally into $50 \mu \mathrm{m}$ thick sections, while supplying DI water to prevent samples from drying out. Bordered pit membranes are covered by a secondary wall having 1 by $5 \mu \mathrm{m}$ elliptical openings. To access and image the pit membrane in AFM, the secondary wall has to be removed without damaging the pit membrane underneath. Longitudinal sectioning sometimes separates from the primary walls leaving intact bordered pit membranes (Figure 1A). As localization of these regions using AFM is exceedingly difficult, the sections were first examined with a 50X optical microscope to locate areas with exposed and undamaged bordered pit membranes. These areas were marked so that they could be located in the AFM. Sections were stored in DI water upon transfer from the light to the AFM microscope. Sample preparation proved to be extremely difficult. Most often the entire process resulted in sections that were unsuitable for AFM scans due to tilt, unexposed or torn bordered pits, problems impossible to detect in light microscope. Thus only a small number of replicates gave useful results despite hundreds of attempts. For visualization purpose only we also used environmental SEM (99\% relative humidity in $0.01 \mathrm{MPa}$ pressure) to image exposed bordered pit membranes prepared in the same way as for AFM analysis.

\section{AFM OBSERVATIONS}

A MFP-3D-BIO ${ }^{\mathrm{TM}}$ Atomic Force Microscope (Asylum Research Inc.) was used in the tapping mode to image hydrated bordered
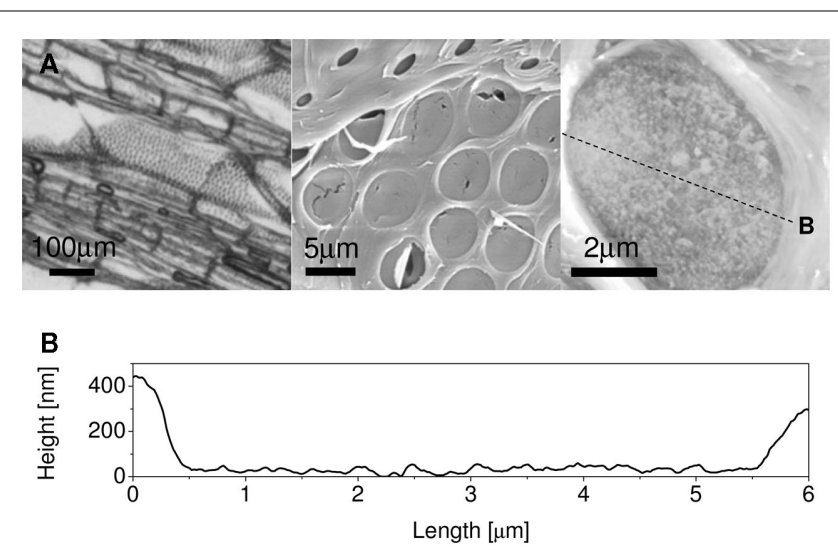

FIGURE 1 | (A) Bordered pit fields imaged with increasing magnification by 50X optical microscope, scanning electron microscope (SEM), and atomic force microscope (AFM). The SEM imaged sample was dried by high vacuum, but both the optical and AFM samples were submerged in DI water. (B) Structural height along the radial direction of the bordered pit membrane imaged by AFM [transect shown as a dashed line in (A)]. pit membranes. Sectioned samples were mounted in the closed fluid cell of the AFM using double-sided tape (3M). Bordered pit samples were submerged and imaged first in DI water followed by imaging in $50 \mathrm{mM} \mathrm{KCl}$ (Sigma Aldrich, NE). The cantilever tip was kept in same position to allow for visualization of pit membrane structure resulting from alterations in the ionic concentration of the liquid medium. The sample and AFM cantilever were aligned using an Olympus $1 \times 81$ inverted microscope (Olympus Inc). A $60 \mu \mathrm{m}$ long silicon nitride bio-lever (Olympus Inc) provided the necessary flexibility for working with submerged samples. Scan rate was $0.3 \mathrm{~Hz}$, around four to five times slower than with dry samples, with scan speed corresponding to the size of the image. The second harmonic resonance frequency was $51.8 \mathrm{kHz}$ and the image resolution was 512 by 512 .

\section{RESULTS}

\section{BORDERED PIT MEMBRANE IN DI WATER}

Atomic force microscopy revealed that the hydrated pit membrane is covered with a relatively disorganized material. Surprisingly, distinct microfibrils, which are usually quite obvious in SEM images (Figure 1A), were not observed. Instead, elevation images reveal a bumpy, but relatively featureless surface across the entire pit membrane (Figure 1B). The roughness of the surface was determined using: $\sqrt{\frac{1}{n} \sum_{n=1}^{n}\left(y_{i}-\bar{y}\right)^{2}}$, where $n$ is the number of analyzed pixels, $y$ is measured height and $\bar{y}$ is the height average. Average roughness was $12.6 \mathrm{~nm}$ with $\mathrm{SD}=2.18$ and $N=10(N$ is the number of independent roughness determinations). The surface of the border pits lacked any visible holes or voids, which are often reported from analysis of dry samples.

The relatively featureless structure of the material covering border pit membranes and lack of the visible cellulosic fibers found on dry imprints of the membranes in SEM studies raises the question of the physical properties of this surface covering. We believe that the microfibrils are hidden inside this material and that under dry conditions the material collapses, behaving as hydrogel. To determine if this hypothesis was true we adjusted the AFM tip position such that the AFM tip penetrated deeper into the covering material. During the taping mode, AFM is designed to keep the position from the sample by feedback control. The position from the surface is determined by the set point, which is typically $80 \%$ of free amplitude (a delicate tap that results in very low tip displacement). To probe deeper into the pit membrane we reduced this set point to $55 \%$. If the covering of the surface was a hard material, the randomness and relative lack of features on the image should remain similar to what was observed using a set point equal to $80 \%$ of free amplitude. Alternatively, the increase of force would result in AFM tip damage (as happen in every case on vessel wall). However, with bordered pits the decrease in free amplitude revealed fiber like structures under the softer cover (Figure 2). The presence of scratch marks across the image may have resulted from material adhering to the tip being dragged along in the scanning direction.

\section{BORDERED PIT MEMBRANE IN KCI}

Replacing DI water with a $50 \mathrm{mM} \mathrm{KCl}$ solution resulted in an immediate and marked change in membrane structure. We chose 


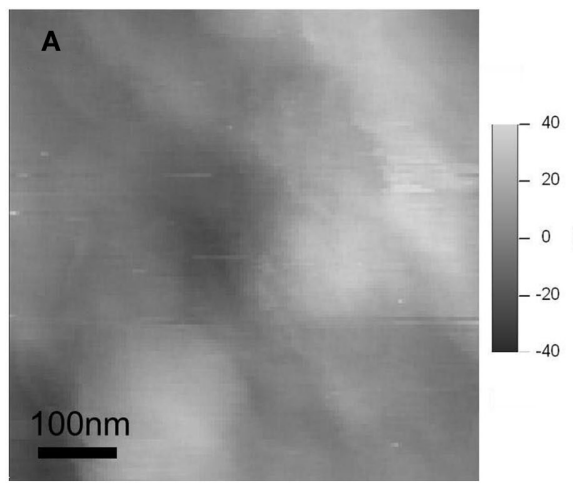

FIGURE 2 | AFM height images with set point of AFM tip position at (A) $\mathbf{8 0} \%$ of free amplitude (B) $\mathbf{5 5 \%}$ of free amplitude. In (B) the AFM tip penetrates the soft material of the bordered pit membrane, exposing the

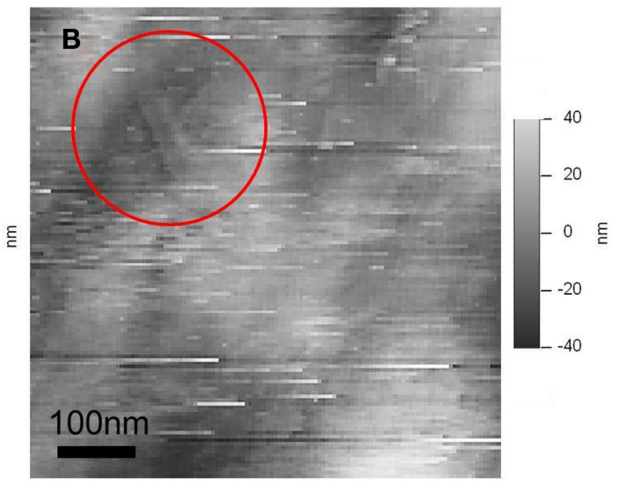

nanofiber structure (seen as straight lines in the red circle). The greater number of scratches in the deeper image $(\mathbf{B})$ is the result of adhesion between AFM tip and the soft matrix.
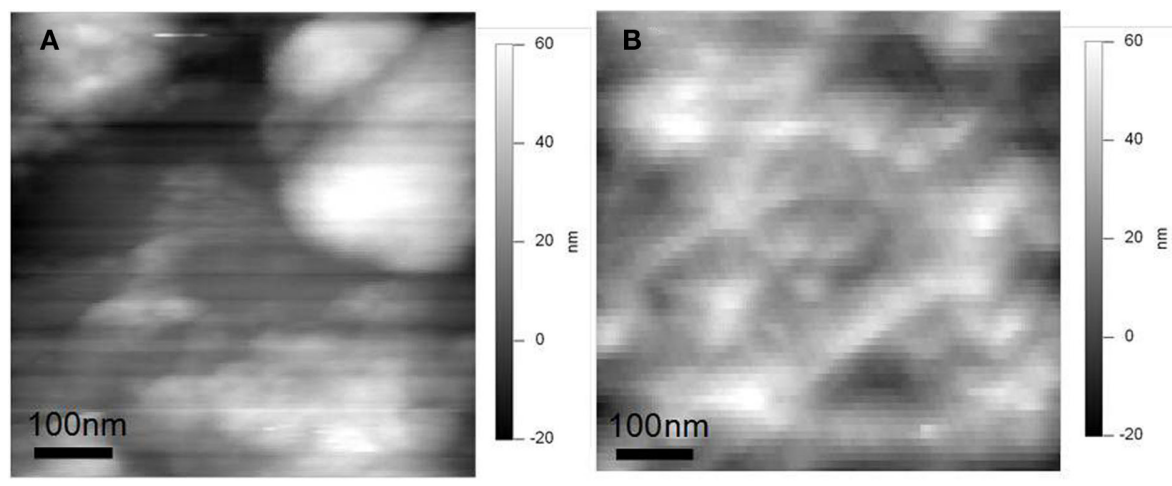

FIGURE 3 | Typical AFM height images with (A) DI water and (B) $\mathbf{5 0 ~} \mathbf{m M ~} \mathbf{~ K C l}$ taken at the same position. Application of $\mathrm{KCl}$ both reduces the height of the border pit membrane and reveals much more structured surface.

$50 \mathrm{mM} \mathrm{KCl}$ based on previous experience that this concentration results in the maximum effect on membrane hydraulic properties (Zwieniecki et al., 2001). Upon exposure to $50 \mathrm{mM} \mathrm{KCl}$, the cantilever tip had to be moved downward around several hundred nanometers to have the same set point. This suggests that the top surface of the bordered pit membrane also moved downward. This drop may indicate the collapse of the soft material. In support of this, the surface was much harder and the microfibrils more exposed (Figure 3). We did not observe the formation of any holes or cracks in the membrane that could have contributed to a lower hydraulic resistance. Instead, the major changes observed included a drop in height which can be interpreted as a decrease in overall membrane thickness (Figure 4) and a significant increase in surface roughness. The average roughness of bordered pit surface increased from 12.6 (in DI water) to $16.6 \mathrm{~nm}$ ( $\mathrm{SD}=1.36$ and $N=10$; in $50 \mathrm{mM} \mathrm{KCl}$ ). This change was significant with $t$-value $=4.94, \mathrm{df}=18$, and $p<0.001$. The increase in mean roughness is consistent with a reduction in membrane thickness. We also observed a change in the signal to noise ratio from 4.2 to 5.5 determined as mean to SD. This change in signal to noise ration was consistent with the presence of harder structures, such as microfibrils, covered by a thinner layer of hydrogel.

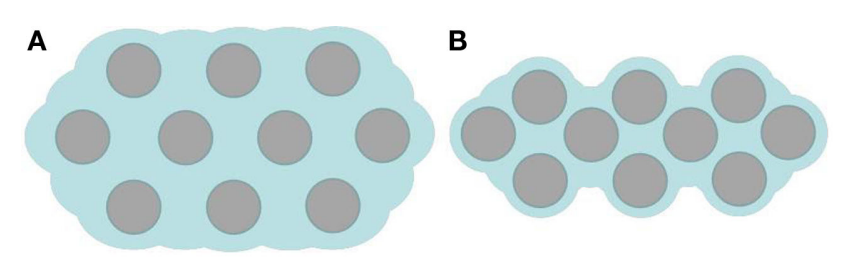

FIGURE 4 | Schematic representation of a bordered pit membrane in cross section (A) before adding $\mathrm{KCl}$ and $(B)$ after adding $\mathrm{KCl}$. The reduction in hydraulic resistance results in part from shortening the water path across the membrane [(gray) microfibril (blue) pectin hydrogel]. Also depicted is the change in surface roughness in $50 \mathrm{mM} \mathrm{KCl}$.

\section{DISCUSSION}

Comparative analysis of pit membrane surface properties in DI water and $50 \mathrm{mM} \mathrm{KCl}$ revealed several important and unexpected properties that enhance our understanding of how water moves through this hybrid material. The current paradigm portrays pit membranes as a microfiber network with discrete holes (Kiss et al., 2009). This view is supported by SEM observations of dry material. In contrast our observation of fully hydrated pit membranes did 
not reveal any holes, but instead a continuous surface of soft material covering fibers with well pronounced elevation features. Upon transfer from DI water to $50 \mathrm{mM} \mathrm{KCl}$, this soft surface underwent a conformational change in its physical properties from soft to hard and from thick to thin, a behavior that is consistent with the properties of hydrogel (Tanaka, 1981). We believe that this observation provides strong support for the notion that bordered pit membranes are composite materials formed from fibrous structure submerged in amorphous gel.

This seemingly simple observation has important implications for our understanding of the hydraulic properties of bordered pit membranes. In particular, it suggests that water does not flow through discrete holes or pores, but instead has to pass through the soft gel (pectin) matrix. Thus, the physical description of water transport across pit membranes in most angiosperms may be similar to what occurs in bio-inspired materials that can sustain tension and operate in a similar manner to xylem or leaves (Noblin et al., 2008; Wheeler and Stroock, 2008). In particular, we may need to change our models for how tension is generated in leaves, as well as how the embolism spreads in the xylem, from ones dominated by surface tension and meniscal failure to ones that emphasize the osmotic forces that can occur within hydrogels.

This study also shifts our understanding of how pit membrane hydraulic permeability is altered in response to ionic strength of xylem sap. Increase in hydraulic conductance may result from changes in membrane thickness (shortening the water path across

\section{REFERENCES}

Beebe, D. J., Moore, J. S., Bauer, J. M., Yu, Q., Liu, R. H., Devadoss, C., and Jo, B. H. (2000). Functional hydrogel structures for autonomous flow control inside microfluidic channels. Nature 404, 588-590.

Choat, B., Brodie, T. W., Cobb, A. R., Zwieniecki, M. A., and Holbrook, N. M. (2006). Direct measurements of intervessel pit membrane hydraulic resistance in two angiosperm tree species. Am. J. Bot. 93, 993-1000.

Cochard, H., Herbette, S., Hernandez, E., Holtta, T., and Mencuccini, M. (2010). The effect of sap ionic composition on xylem vulnerability to cavitation. J. Exp. Bot. 61, 275-285.

Dute, R. R., and Elder, T. (2011). Atomic Force microscopy of torus-bearing pit membranes. Iawa J. 32, 415-430.

Holbrook, N. M., and Zwieniecki, M. A. (2005). Embolism repair. Comp. Biochem. Physiol. Part A Mol. Integr. Physiol. 141, S302-S302.

Jansen, S., Choat, B., and Pletsers, A. (2009). Morphological variation of intervessel pit membranes and implications to xylem function in angiosperms. Am. J. Bot. 96, 409-419.

Kirk, S., Skepper, J., and Donald, A. M. (2009). Application of environmental scanning electron

the membrane) and changes in permeability of the charged hydrogel (Mattern et al., 2008), while previously proposed changes in the dimension of channels might be less influential (Zwieniecki et al., 2001). This finding underscores the role of pit membranes in protection from embolism as ionic strength does not necessarily decrease the pressure threshold for the air to pass from one vessel to another (Cochard et al., 2010). In addition, the observed composite nature of the pit membrane explains their ability to recover from mechanical damage (Sperry and Hacke, 2004), as changes in gel hydration allow cellulose microfibrils to shift within the network and thus prevent the formation of permanent holes or cracks. Such healing properties are especially important for plants that sustain multiple cycles of embolism and embolism removal during drought stress (Sperry and Hacke, 2004). The conformation response of pit membranes to variation in ions reported here suggests that we need to replace the current emphasis on the structural framework provided by the cellulose microfibrils to flow models dominated by the properties of the gel matrix (Wheeler and Stroock, 2008).

\section{ACKNOWLEDGMENTS}

It is a pleasure to thank Dr. Jiangdong Deng at Center for Nanoscale System in Harvard University and Dr. Hongsik Park at Brown University for many useful discussions of advanced use for AFM. This work was supported by the Materials Research Science and Engineering Center at Harvard University and a grant from the Air Force Office of Sponsored Research.

sebiferum (Euphorbiaceae). IAWA J. 26, 397-426.

Shackel, K. A., Brodersen, C. R., McElrone, A. J., Choat, B., and Matthews, M. A. (2010). The dynamics of embolism repair in xylem: in vivo visualizations using high-resolution computed tomography. Plant Physiol. 154, 1088-1095.

Sperry, J. S., and Hacke, U. G. (2004) Analysis of circular bordered pit function - I. Angiosperm vessels with homogenous pit membranes. Am. J. Bot. 91, 369-385.

Tanaka, T. (1981). Gels. Sci. Am. 244, 124-138.

Vyawahare, S., Sitaula, S., Martin, S., Adalian, D., and Scherer, A. (2008). Electronic control of elastomeric microfluidic circuits with shape memory actuators. Lab. Chip 8, 1530-1535.

Wheeler, T. D., and Stroock, A. D. (2008). The transpiration of water at negative pressures in a synthetic tree. Nature 455, 208-212.

Zwieniecki, M. A., and Holbrook, N. M. (2000). Bordered pit structure and vessel wall surface properties. Implications for embolism repair. Plant Physiol. 123, 1015-1020.

Zwieniecki, M. A., and Holbrook, N. M. (2009). Confronting Maxwell's demon: biophysics of xylem embolism repair. Trends Plant Sci. 14, 530-534.

Zwieniecki, M. A., Melcher, P. J., and Holbrook, N. M. (2001). Hydrogel control of xylem hydraulic resistance in plants. Science 291, 1059-1062.

Conflict of Interest Statement: The authors declare that the research was conducted in the absence of any commercial or financial relationships that could be construed as a potential conflict of interest.

Received: 02 November 2011; paper pending published: 18 November 2011; accepted: 01 March 2012; published online: 21 March 2012.

Citation: Lee J, Holbrook NM and Zwieniecki MA (2012) Ion induced changes in the structure of bordered pit membranes. Front. Plant Sci. 3:55. doi: 10.3389/fpls.2012.00055

This article was submitted to Frontiers in Plant Biophysics and Modeling, a specialty of Frontiers in Plant Science.

Copyright (c) 2012 Lee, Holbrook and Zwieniecki. This is an open-access article distributed under the terms of the Creative Commons Attribution Non Commercial License, which permits noncommercial use, distribution, and reproduction in other forums, provided the original authors and source are credited. 